\title{
Formation Tracking for Nonaffine Nonlinear Multiagent Systems Using Neural Network Adaptive Control
}

\author{
Tongjuan Zhao $\mathbb{D}^{1,2}$ Jiuhe Wang $\mathbb{D},{ }^{1}$ and Jianhua Zhang $\mathbb{D}^{3}$ \\ ${ }^{1}$ Yanshan University, Qinhuangdao, Hebei 066004, China \\ ${ }^{2}$ Qinhuangdao Vocational and Technical College, Qinhuangdao, Hebei 066100, China \\ ${ }^{3}$ School of Information and Control Engineering, Qingdao University of Technology, Qingdao, Shandong 266525, China
}

Correspondence should be addressed to Jianhua Zhang; jianhuazhang@aliyun.com

Received 8 May 2020; Revised 29 September 2020; Accepted 13 October 2020; Published 26 October 2020

Academic Editor: Olfa Boubaker

Copyright (c) 2020 Tongjuan Zhao et al. This is an open access article distributed under the Creative Commons Attribution License, which permits unrestricted use, distribution, and reproduction in any medium, provided the original work is properly cited.

Adaptive tracking control for distributed multiagent systems in nonaffine form is considered in this paper. Each follower agent is modeled by a nonlinear pure-feedback system with nonaffine form, and a nonlinear system is unknown functions rather than constants. Radial basis function neural networks (NNs) are employed to approximate the unknown nonlinear functions, and weights of NNs are updated by adaptive law in finite-time form. Then, the adaptive finite NN approach and backstepping technology are combined to construct the consensus tracking control protocol. Numerical simulation is presented to demonstrate the efficacy of suggested control proposal.

\section{Introduction}

Due to the limitations of a single agent in completing some special tasks and the needs of human society, the multiagent system has received extensive attention from the academic engineering community. In recent years, distributed information processing technology has been widely used, such as urban transportation [1], intelligent robots [2], flexible manufacturing [3], and coordinated expert systems [4] due to higher fault tolerance and reliability. It has attracted the attention of experts and scholars in different fields such as artificial intelligence and control engineering.

In the 1980s, multiagent systems were researched and applied, and in recent years, it has become one of the hot spots in the field of artificial intelligence. For multiagent systems, the consensus problem is the most basic control problem; see a large number of academic papers [5-7]. The key to consensus is to design a reasonable consensus protocol to ensure that the multiagent system is consistent. Scholars have further explored multiagent systems with different structures, such as directed topography and undirected topography [8-10]. However, it is difficult to avoid the interference factors of uncertainty, thus applying robust consensus control [11-13]. In recent years, due to the inevitable interference factors, the robust consensus problem has received widespread attention and received widespread attention. In [14], the robust consensus control of the Laplacian matrix uncertainty multiagent system is studied, and the conditions for achieving state consensus are proposed. In [15], the robust consensus of linear multiagents with random switching topology was studied. In [16], the second-order robustness of nonlinear multiagents with extended state observers is studied. In [17], scholars studied the distributed robust adaptive consensus control of uncertain nonlinear fractional multiagent systems. As we all know, finite-time convergence is a topic that has attracted much attention and is of great significance. Therefore, some scholars have raised the problem of finite-time consensus [18-20]. Some scholars consider the issue of fixed-time consensus.

In recent years, consensus-tracking problem of distributed multiagent systems has broad applications in many areas, such as formation control, flocking, cooperative control of UAV, and distributed sensor networks [21, 22]. Formation tracking control originated from various natural phenomena, such as flocking in birds, fish, and so on. There 
exist some kinds of consensus problems of multiagent systems, because sometimes it is needed that all agents agree on some desired quantity of interest, sometimes not. In the leader follower multiagent systems, consensus means that all the follower agents reach the leader values in a finite time $[23,24]$.

Recently, finite-time stability has received much attention due to its efficient performance in many areas $[25,26]$. Especial neural networks control is a powerful control method, because neural networks can approximate nonlinear system without model $[27,28]$. Though Lyapunov uniformly ultimately bounded (UUB) results solve some nonlinear system control problem, both bounded and exponential convergence speed cause confusion. It should be noted that the research on finite-time neural network control is still in a very beginning stage. The key issue is how to systematically obtain finite-time adaptive law of neural network weight from finite-time convergence of closed-loop systems. In regarding to such neural network-based adaptive control to the authors' best knowledge, there are a few results about finite-time adaptive neural network control because it is not easy to design the finite-time neural network adaptive controller, and there exists lack of relevant inequality skills to finish finite-time stability analysis. There has been any reference to show finite-time adaptive algorithm for weights of NNs having been expanded to solve the problems of finite control for pure-feedback nonaffine nonlinear systems.

The remainder of this paper is organized as follows. The multiagent system is described in Section 2. The proposed algorithms for formation tracking, based on the finite-time adaptive control, are presented in Section 3. Theoretical analysis of the model is given in Section 4. Simulation results are given in Section 5. Finally, conclusions are drawn in Section 6 .

\section{Problem Description}

2.1. System Description. Consider a class of the nonlinear pure-feedback multiagent system, composed of $N$ follower agents (labeled from 1 to $N$ ), and a leader (labeled $d$ ). The communication topology of followers are described by a digraph $G$. The dynamic model of the $i$ th $(i=1,2, \ldots, N)$ follower is

$$
\left\{\begin{array}{l}
\dot{x}_{i, k}=f_{i, k}\left(\bar{x}_{i, k+1}\right), \quad 1 \leq k \leq n_{i}-1, \\
\dot{x}_{i, n_{i}}=f_{i, n_{i}}\left(\bar{x}_{i, n_{i}}, u_{i}\right), \\
y_{i}=x_{i, 1}
\end{array}\right.
$$

where $\bar{x}_{i, j}=\left[x_{i, 1}, x_{i, 2}, \ldots, x_{i, j}\right]^{T} \in \Re^{j}, \bar{x}_{i, n_{i}}=\left[x_{i, 1}, x_{i, 2}, \ldots\right.$, $\left.x_{i, n_{i}}\right]^{T} \in \mathfrak{R}^{n_{i}}$ is the entire state variables of the $i$ th agent, $u_{i} \in \Re, y_{i} \in \mathfrak{R}, \varphi_{i}(t)$ indicate the state, control, output, and initial condition, respectively, and, $f_{i, k}(\cdot)$ are nonlinear smooth functions.

2.2. Algebraic Graph Theory. A directed graph is represented by $G=(V, E)$ with $E \subseteq V \times V$ denoting edge set and $V=$ $\left\{v_{1}, v_{2}, \ldots, v_{k}\right\}$ is the node set. An edge $e_{j i}=\left(v_{j}, v_{i}\right) \in E$ of the graph $G$ means that $i$ can get messages from $j$; meanwhile, it is also said that the agent $j$ is one of agent $i$ 's neighbors, not vice versa.

Hence, the agent $i$ 's neighbor set is $N_{i}=\left\{v_{j} \mid\left(v_{j}, v_{i}\right) \in E\right\}$. When weight of edges is considered, the graph is said to be a weighted graph. $A=\left[a_{i j}\right] \in \mathfrak{R}^{k \times k}$ (adjacency matrix) is often used to express the graphic topology. For the element $a_{i j}$, it is defined that $a_{i j}>0$ if $e_{j i}=\left(v_{j}, v_{i}\right) \in E$, otherwise, $a_{i j}=0$. Self-loop is not considered as usual, i.e., $a_{i i}=0$, and $D=$ $\operatorname{diag}\left(d_{1}, d_{2}, \ldots, d_{k}\right) \in \mathfrak{R}^{k \times k}$ is defined as an in-degree matrix, where $d_{i}=\sum_{j=1}^{k} a_{i j}$ of $D$ is in-degree for agent $i$. The row sum of Laplacian matrix $L=D-A$ is 0 and $L 1=0$. A directed graph is said to have direct path means that there exists an edge sequence in the form of $\left\{\left(v_{i}, v_{r}\right),\left(v_{r}\right.\right.$, $\left.\left.v_{s}\right), \ldots,\left(v_{t}, v_{j}\right)\right\}$. When there exists at least one agent (root) in the digraph which can transmit information through direct path to all other agents, the direct graph is thus said to include a directed spanning tree. The local tracking error for agent $i$ can be described as

$$
z_{i, 1}=\sum_{j=1}^{N} a_{i j}\left(y_{i}-y_{j}\right)+b_{i}\left(y_{i}-y_{d}\right),
$$

where $i=1,2, \ldots, N$, the pinning gain $b_{i} \geq 0$, where $b_{i}>0$ denotes the weight between the $i$ th agent and leader agent.

2.3. Radial Basis Function Neural Networks (RBFNNs) and Function Approximation. In brief, the following radial basis function (RBF) NN is used to approximate the continuous function $F(x): \mathfrak{R}^{n} \longrightarrow \mathfrak{R}$ over a compact set

$$
F_{N N}(x, W)=W^{T} \Psi(x),
$$

where input $x \in \Omega \subset \Re^{n}$, weight vector $W=\left[w_{1} \ldots\right.$ $\left.w_{l}\right]^{T} \in \mathfrak{R}^{l}$, and node vector $\Psi(x)=\left[\psi_{1}(x) \ldots \psi_{l}(x)\right]^{T}$, in which the element $\psi_{i}(x)$ is being chosen as the commonly used Gaussian function as follows:

$$
\psi_{i}(x)=\exp \left[\frac{-\left(x-\mu_{i}\right)^{T}\left(x-\mu_{i}\right)}{\eta_{i}^{2}}\right], \quad i=1,2, \ldots, l,
$$

where $\mu_{i}=\left[\mu_{i 1} \ldots \mu_{i n}\right]^{T}$ is the center of the receptive field and $\eta_{i}$ is the width of the Gaussian function.

It has been proven that RBF NN can approximate any continuous function over a compact set $\Omega_{x} \subset \Re^{n}$ as

$$
F(x)=W^{* T} \Psi(x)+\varepsilon(x),
$$

where $W^{*}$ is the ideal NN weight and $\varepsilon(x)$ is the NN approximation error:

$$
W^{*}=\arg \min _{W \in \mathfrak{R}^{l}}\left\{\sup \left|F(x)-W^{T} \Psi(x)\right|\right\} .
$$

Notation: throughout this paper, $W \in \mathfrak{R}^{m \times n}$ represents the matrix, $W^{*}, \widehat{W}, \widetilde{W}$ indicate ideal weight, estimated weight, and error between ideal and estimated weight. Throughout this paper, $W \in \mathfrak{R}^{m \times n}$ represents the matrix, $W=\left[w_{i j}\right]_{m \times n}$, rational number $\eta$ and matrix, matrix $W^{\eta}$ denote element-by-element powers $W^{\eta}=\left[w_{i j}^{\eta}\right]_{m \times n}$, and $\widetilde{W}^{\eta T}$ denote the transposition of matrix $\widetilde{W}^{\eta}$, such as 
$\widetilde{W}^{\eta T}=\left(\tilde{W}^{\eta}\right)^{T} \cdot W^{*}, W, \widetilde{W}$ indicate ideal weight, estimated weight, and error between ideal and estimated weight.

Definition 1. Consider the system $\dot{x}=f(x, u)$, where $x$ is a state vector and $u$ is the input vector. The solution is practical finite stable (PFS) if for all $x\left(t_{0}\right)=x_{0}$, there exists $\varepsilon>0$ and $T\left(\varepsilon, x_{0}\right)<\infty$, such that $\|x\|<\varepsilon$, for all $t \geq t_{0}+T$.

Lemma 1. Consider the system $\dot{x}=f(x, u)$, and suppose that there exist continuous function $V(x)$, scalars $\lambda>0,0<\alpha<1$, and $0<\eta<\infty$ such that

$$
\dot{V}(x) \leq-\lambda V^{\alpha}(x)+\eta \text {. }
$$

Then, the trajectory of the system $\dot{x}=f(x, u)$ is PFS.

Lemma 2. Young's inequality: for any constant $a, b \in \mathfrak{R}$, the following inequality holds:

$$
a b \leq \frac{1}{p} a^{p}+\frac{1}{q} b^{q} .
$$

where $p>1, q>1$, and $(1 / p)+(1 / q)=1$.

Remark 1. Based on Young's inequality, then the following inequalities hold:

$$
\begin{aligned}
z \varepsilon & \leq \frac{3}{4} z^{4 / 3}+\frac{1}{4} \mathcal{E}^{4} \\
-\widetilde{W}^{T} \widehat{W}^{1 / 3} & \leq-\frac{1}{2} \widetilde{W}^{(2 / 3) T} \widetilde{W}^{2 / 3}+W^{*(2 / 3) T} W^{*(2 / 3)}
\end{aligned}
$$

\section{Distributed Adaptive Tracking Controller Design}

Consider system (1) and tracking error (2), and define

$$
\begin{aligned}
z_{i, k} & =x_{i, k}-\alpha_{i, k-1}, \quad k=2,3, \ldots, n_{i}, \\
z_{i, n_{i}+1} & =u_{i}-\alpha_{i, n_{i}},
\end{aligned}
$$

where $\alpha_{i, j},\left(2 \leq j \leq n_{i}\right)$ is the virtual control; in the first step, consider the system $z_{i, 1}$.

Then, it has

$$
\begin{aligned}
\dot{z}_{i, 1} & =\left(\sum_{j=1}^{N} a_{i j}+b_{i}\right) \dot{x}_{i, 1}-\sum_{j=1}^{N} a_{i j} \dot{x}_{j, 1}-b_{i} f_{d}\left(x_{d}, t\right) \\
& =\left(\sum_{j=1}^{N} a_{i j}+b_{i}\right) f_{i, 1}\left(\bar{x}_{i, 2}\right)-\sum_{j=1}^{N} a_{i j} f_{j, 2}\left(\bar{x}_{j, 2}\right)-b_{i} f_{d}\left(x_{d}, t\right) .
\end{aligned}
$$

Base on ideal virtual control law, and choose the NNs to approximate the nonlinear system

$$
\left(\sum_{j=1}^{N} a_{i j}+b_{i}\right) f_{i, 1}\left(\bar{x}_{i, 2}\right)-\sum_{j=1}^{N} a_{i j} f_{j, 2}\left(\bar{x}_{j, 2}\right)-b_{i} f_{d}\left(x_{d}, t\right)=W_{i, 1}^{T *} \Psi_{1}\left(Z_{2}\right)+\varepsilon_{i, 1} .
$$

Therefore,

$$
\dot{z}_{i, 1}=W_{i, 1}^{* T} \Psi_{1}\left(Z_{2}\right)+\varepsilon_{i, 1}
$$

Choose the practical virtual control law

$$
\alpha_{i, 1}=-k_{1} z_{i, 1}^{1 / 3}+x_{i, 2}-\widehat{W}_{1}^{T} \Psi_{1}\left(Z_{2}\right) .
$$

Choose the adaptive law

$$
\dot{\widehat{W}}_{i, 1}=\Gamma_{i, 1}\left[z_{i, 1} \Psi_{1}\left(Z_{2}\right)-\sigma_{i, 1} \widehat{W}_{i, 1}^{1 / 3}\right]
$$

where $\Gamma_{i, 1}=\Gamma_{i, 1}^{T}>0$, and $\sigma_{i, 1}>0$ is positive constant design parameters.

Then, based on (12) and (13),

$$
\dot{z}_{i, 1}=W_{i, 1}^{* T} \Psi_{1}\left(Z_{2}\right)+\varepsilon_{i, 1}-k_{i, 1} z_{i, 1}^{1 / 3}+x_{i, 2}-\alpha_{i, 1}-\widehat{W}_{i, 1}^{T} \Psi_{1}\left(Z_{2}\right)
$$

Let

$$
z_{i, 2}=x_{i, 2}-\alpha_{i, 1}
$$

Therefore,

$$
\dot{z}_{i, 1}=-\tilde{W}_{i, 1}^{T} \Psi_{1}\left(Z_{2}\right)-k_{i, 1} z_{i, 1}^{1 / 3}+z_{i, 2}+\varepsilon_{i, 1}
$$

where

$$
\widetilde{W}_{i, 1}=\widehat{W}_{i, 1}-W_{i, 1}^{*}
$$

Choose the Lyapunov candidate function

$$
V_{i, 1}=\frac{1}{2} z_{i, 1}^{2}+\frac{1}{2} \widetilde{W}_{i, 1}^{T} \Gamma_{i, 1}^{-1} \widetilde{W}_{i, 1} \text {. }
$$

Then,

$$
\begin{aligned}
\dot{V}_{i, 1} & =z_{i, 1} \dot{z}_{i, 1}+\widetilde{W}_{i, 1}^{T} \Gamma_{i, 1}^{-1} \dot{\tilde{W}}_{i, 1} \\
& =-k_{i, 1} z_{i, 1}^{4 / 3}+z_{i, 1} z_{i, 2}+z_{i, 1} \varepsilon_{i, 1}-\sigma_{i, 1} \widetilde{W}_{i, 1}^{T} \widehat{W}_{i, 1}^{1 / 3} .
\end{aligned}
$$

Based on based inequalities, the following holds: 


$$
\begin{aligned}
z_{i, 1} \varepsilon_{i, 1} & \leq \frac{3}{4} z_{i, 1}^{4 / 3}+\frac{1}{4} \varepsilon_{i, 1}^{4}-\sigma_{i, 1} \widehat{W}_{i, 1}^{T} \widehat{W}_{i, 1}^{1 / 3} \\
& \leq-\frac{\sigma_{1}}{2} \widetilde{W}_{i, 1}^{(2 / 3) T} \widetilde{W}_{i, 1}^{2 / 3}+\sigma_{1} W_{i, 1}^{(2 / 3) * T} W_{i, 1}^{2 / 3 *}
\end{aligned}
$$

Then, based on (21), it gives

$$
\begin{aligned}
\dot{V}_{i, 1}= & -\left(k_{i, 1}-\frac{3}{4}\right) z_{i, 1}^{4 / 3}+z_{i, 1} z_{i, 2}-\frac{\sigma_{i, 1}}{2} \widetilde{W}_{i, 1}^{(2 / 3) T} \tilde{W}_{i, 1}^{2 / 3} \\
& +\frac{1}{4} \varepsilon_{i, 1}^{4}+\sigma_{i, 1} W_{i, 1}^{(2 / 3) * T} W_{i, 1}^{2 / 3 *} .
\end{aligned}
$$

Then, it has

$$
\begin{aligned}
\dot{V}_{i, 1} \leq & z_{i, 1} z_{i, 2}-\eta_{i, 1}\left(\frac{z_{i, 1}^{2}}{2}\right)^{2 / 3}-\frac{\eta_{i, 1}}{2}\left(\frac{\widetilde{W}_{i, 1}^{\mathrm{T}} \Gamma_{i, 1}^{-1} \widetilde{W}_{i, 1}}{2}\right)^{2 / 3} \\
& +\frac{1}{4} \varepsilon_{i, 1}^{4}+\sigma_{i, 1} W_{i, 1}^{(2 / 3) * T} W_{i, 1}^{(2 / 3) *} \leq z_{i, 1} z_{i, 2}-\eta_{i, 1} V_{i, 1}^{2 / 3}+\delta_{i, 1},
\end{aligned}
$$

where

$$
\begin{aligned}
& \eta_{i, 1}=\min \left(4^{1 / 3}\left(k_{i, 1}-\frac{3}{4}\right), \frac{\sigma_{i, 1}}{2^{1 / 3} \lambda_{\max }\left(\Gamma_{i, 1}^{-1}\right)^{2 / 3}}\right), \\
& \delta_{i, 1}=\frac{1}{4} \varepsilon_{i, 1}^{4}+\sigma_{i, 1} W_{i, 1}^{(2 / 3) * T} W_{i, 1}^{(2 / 3) *} .
\end{aligned}
$$

The $j$ th step $2 \leq j \leq n_{i}$ :

$$
z_{i, j}=x_{i, j}-\alpha_{i, j-1}
$$

And $x_{i, j+1}=u_{i}, \dot{u}_{i}=v_{i}$, then choose the virtual control law, and choose the $\mathrm{NN}$ to approximate the nonlinear system $\dot{x}_{i, j}-\dot{\alpha}_{i, j-1}$

$$
f_{i, j}\left(\bar{x}_{i, j+1}\right)-\dot{\alpha}_{i, j-1}=W_{i, j}^{* T} \Psi_{j}\left(Z_{j+1}\right)+\varepsilon_{i, j} .
$$

Based on the system,

$$
\dot{z}_{i, j}=W_{i, j}^{* T} \Psi_{j}\left(Z_{j+1}\right)+\varepsilon_{i, j}
$$

Choose the practical virtual control law

$$
\alpha_{i, j}=-z_{i, j-1}-k_{i, j} z_{i, j}^{1 / 3}+x_{i, j+1}-\widehat{W}_{i, j}^{T} \Psi_{j}\left(Z_{j+1}\right) .
$$

Choose the adaptive law

$$
\dot{\widehat{W}}_{i, j}=\Gamma_{i, j}\left[z_{i, j} \Psi_{j}\left(Z_{j+1}\right)-\sigma_{i, j} \widehat{W}_{i, j}^{1 / 3}\right],
$$

where $\Gamma_{i, j}=\Gamma_{i, j}^{T}>0$, and $\sigma_{i, j}>0$ is positive constant design parameters. Then,

$$
\dot{z}_{i, j}=-\widetilde{W}_{i, j} \Psi_{j}\left(Z_{j+1}\right)-z_{i, j-1}-k_{i, j} z_{i, j}^{1 / 3}+z_{i, j+1}+\varepsilon_{i, j},
$$

where

$$
\widetilde{W}_{i, j}=\widehat{W}_{i, j}-W_{i, j}^{*}
$$

Choose the Lyapunov candidate function

$$
V_{i, j}=\frac{1}{2} z_{i, j}^{2}+\frac{1}{2} \widetilde{W}_{i, j}^{T} \Gamma_{i, j}^{-1} \widetilde{W}_{i, j}
$$

Then,

$$
\begin{aligned}
\dot{V}_{i, j} & =z_{i, j} \dot{z}_{i, j}+\widetilde{W}_{i, j} \Gamma_{i, j}^{-1} \dot{\tilde{W}}_{i, j}^{T} \\
& =-z_{i, j-1} z_{i, j}-k_{i, j} z_{i, j}^{4 / 3}+z_{i, j} z_{i, j+1}+z_{i, j} \varepsilon_{i, j}-\sigma_{i, j} \widetilde{W}_{i, j}^{T} \widehat{W}_{i, j}^{1 / 3} .
\end{aligned}
$$

Based on basic equation, the following inequalities hold:

$$
\begin{aligned}
z_{i, j} \varepsilon_{i, j} & \leq \frac{3}{4} z_{i, j}^{4 / 3}+\frac{1}{4} \varepsilon_{i, j}^{4}-\sigma_{i, j} \widehat{W}_{i, j}^{T} \widehat{W}_{i, j}^{1 / 3} \\
& \leq-\frac{\sigma_{i, j}}{2} \widehat{W}_{i, j}^{(2 / 3) T} \tilde{W}_{i, j}^{2 / 3}+\sigma_{i, j} W_{i, j}^{*(2 / 3) T} W_{i, j}^{*(2 / 3)} .
\end{aligned}
$$

Then, based on (33), it yields

$$
\begin{aligned}
\dot{V}_{i, j}= & z_{i, j} \dot{z}_{i, j}+\tilde{W}_{i, j}^{T} \Gamma_{i, j}^{-1} \dot{\tilde{W}}_{i, j} \\
= & -z_{i, j-1} z_{i, j}-\left(k_{i, j}-\frac{3}{4}\right) z_{i, j}^{4 / 3}+z_{i, j} z_{i, j+1} \\
& -\frac{\sigma_{i, j}}{2} \tilde{W}_{i, j}^{(2 / 3) T} \tilde{W}_{i, j}^{2 / 3}+\frac{1}{4} \varepsilon_{i, j}^{4}+\sigma_{i, j} W_{i, j}^{(2 / 3) * T} W_{i, j}^{(2 / 3) *} .
\end{aligned}
$$

Continue

$$
\begin{aligned}
\dot{V}_{i, j} \leq & -z_{i, j-1} z_{i, j}+z_{i, j} z_{i, j+1}-\eta_{i, j}\left(\frac{z_{i, j}^{2}}{2}\right)^{2 / 3} \\
& -\eta_{i, j}\left(\frac{\tilde{W}_{i, j}^{T} \Gamma_{i, j}^{-1} \tilde{W}_{i, j}}{2}\right)^{2 / 3}+\frac{1}{4} \varepsilon_{i, j}^{4}+\sigma_{i, j} W_{i, j}^{(2 / 3) * T} W_{i, j}^{(2 / 3) *} \\
\leq & -z_{i, j-1} z_{i, j}+z_{i, j} z_{i, j+1}-\eta_{i, j} V_{i, j}^{2 / 3}+\delta_{i, j},
\end{aligned}
$$

where

$$
\begin{aligned}
& \eta_{i, j}=\min \left(4^{1 / 3}\left(k_{i, j}-\frac{3}{4}\right), \frac{\sigma_{i, j}}{2^{1 / 3} \lambda_{\max }\left(\Gamma_{i, j}^{-1}\right)^{2 / 3}}\right), \\
& \delta_{i, j}=\frac{1}{4} \varepsilon_{i, j}^{4}+\sigma_{i, j} W_{i, j}^{(2 / 3) * T} W_{i, j}^{(2 / 3) *} .
\end{aligned}
$$

The $n$th step is the most important step. Based on the system, 


$$
z_{i, n_{i}}=x_{i, n_{i}}-\alpha_{i, n_{i}-1} .
$$

Choose the NN to approximate the nonlinear system

$$
f_{i, n_{i}}\left(\bar{x}_{i, n_{i}}, u_{i}\right)-\dot{\alpha}_{i, n_{i}-1}=W_{i, n_{i}}^{* T} \Psi_{n_{i}}\left(Z_{n_{i}+1}\right)+\varepsilon_{i, n_{i}} .
$$

Based on the system,

$$
\dot{z}_{i, n_{i}}=W_{i, n_{i}}^{* T} \Psi_{n_{i}}\left(Z_{n_{i}+1}\right)+\varepsilon_{i, n_{i}} .
$$

Choose practical virtual control law

$$
\alpha_{i, n_{i}}=-z_{i, n_{i}-1}-k_{i, n_{i}} z_{i, n_{i}}+u_{i}-\widehat{W}_{i, n_{i}}^{T} \Psi_{n_{i}}\left(Z_{n_{i}+1}\right) .
$$

Choose adaptive law

$$
\dot{\widehat{W}}_{i, n_{i}}=\Gamma_{i, n_{i}}\left[z_{i, n_{i}} \Psi_{n_{i}}^{T}\left(Z_{n_{i}+1}\right)-\sigma_{i, n_{i}} \widehat{W}_{n_{i}}^{1 / 3}\right],
$$

where $\Gamma_{i, n_{i}}=\Gamma_{i, n_{i}}^{T}>0$, and $\sigma_{i, n_{i}}>0$ are positive constant design parameters; then,

$$
\dot{z}_{i, n_{i}}=-\widetilde{W}_{i, n_{i}}^{T} \Psi_{n_{i}}\left(Z_{n_{i}+1}(t)\right)-z_{i, n_{i}-1}-k_{i, n_{i}} z_{i, n_{i}}^{1 / 3}+z_{i, n_{i}+1}+\varepsilon_{i, n_{i}},
$$

where

$$
\widetilde{W}_{i, n_{i}}=\widehat{W}_{i, n_{i}}-W_{i, n_{i}}^{*} .
$$

From the following inequality,

$$
\begin{aligned}
-\sigma_{i, n_{i}} \widetilde{W}_{i, n_{i}}^{T} \widehat{W}_{i, n_{i}}^{1 / 3} \leq & -\frac{\sigma_{i, n_{i}}}{2} \widetilde{W}_{i, n_{i}}^{(2 / 3) T} \tilde{W}_{i, n_{i}}^{2 / 3} \\
& +\sigma_{i, n_{i}} W_{i, n_{i}}^{(2 / 3) * T} W_{i, n_{i}}^{(2 / 3) *} .
\end{aligned}
$$

Choose the Lyapunov candidate function

$$
V_{i, n_{i}}=\frac{1}{2} z_{i, n_{i}}^{2}+\frac{1}{2} \tilde{W}_{i, n_{i}} \Gamma_{i, n_{i}}^{-1} \tilde{W}_{i, n_{i}}^{T}
$$

Then,

$$
\begin{aligned}
\dot{V}_{i, n_{i}}= & z_{i, n_{i}} \dot{z}_{i, n_{i}}+\widetilde{W}_{i, n_{i}} \Gamma_{i, n_{i}}^{-1} \dot{\tilde{W}}_{i, n_{i}}^{T} \\
= & -z_{i, n_{i}-1} z_{i, n_{i}}-k_{i, n_{i}} z_{i, n_{i}}^{4 / 3}+z_{i, n_{i}} z_{i, n_{i}+1} \\
& +z_{i, n_{i}} \varepsilon_{i, n_{i}}-\sigma_{i, n_{i}} \widetilde{W}_{i, n_{i}}^{T} \widehat{W}_{i, n_{i}}^{1 / 3} .
\end{aligned}
$$

Based on Lemma, the following inequalities hold:

$$
\begin{aligned}
z_{i, j} \varepsilon_{i, n_{i}} & \leq \frac{3}{4} z_{i, n_{i}}^{4 / 3}+\frac{1}{4} \varepsilon_{i, n_{i}}^{4}-\sigma_{i, n_{i}} \widetilde{W}_{i, n_{i}}^{T} \widehat{W}_{i, n_{i}}^{1 / 3} \\
& \leq-\frac{\sigma_{i, n_{i}}}{2} \widetilde{W}_{i, n_{i}}^{(2 / 3) T} \widetilde{W}_{i, n_{i}}^{2 / 3}+\sigma_{i, n_{i}} W_{i, n_{i}}^{*(2 / 3) T} W_{i, n_{i}}^{*(2 / 3)} .
\end{aligned}
$$

Then, it gives

$$
\begin{aligned}
\dot{V}_{i, n_{i}} \leq & -z_{i, n_{i}-1} z_{i, n_{i}}-\left(k_{i, n_{i}}-\frac{3}{4}\right) z_{i, n_{i}}^{4 / 3}+z_{i, n_{i}} z_{i, n_{i}+1} \\
& -\frac{\sigma_{i, n_{i}}}{2} \tilde{W}_{i, n_{i}}^{(2 / 3) T} \tilde{W}_{i, n_{i}}^{2 / 3}+\frac{1}{4} \varepsilon_{i, n_{i}}^{4}+\sigma_{i, n_{i}} W_{i, n_{i}}^{(2 / 3) * T} W_{i, n_{i}}^{(2 / 3) *} .
\end{aligned}
$$

Then,

$$
\begin{aligned}
\dot{V}_{i, n_{i}} \leq & -z_{i, n_{i}-1} z_{i, n_{i}}+z_{i, n_{i}} z_{i, n_{i}+1}-\eta_{i, n_{i}}\left(\frac{z_{i, n_{i}}^{2}}{2}\right)^{2 / 3} \\
& -\eta_{i, n_{i}}\left(\frac{\widetilde{W}_{i, n_{i}}^{T} \Gamma_{i, n_{i}}^{-1} \tilde{W}_{i, n_{i}}}{2}\right)^{2 / 3}+\delta_{i, n_{i}} \\
\leq & -z_{i, n_{i}-1} z_{i, n_{i}}+z_{i, n_{i}} z_{i, n_{i}+1}-\eta_{i, n_{i}} V_{i, n_{i}}^{2 / 3}+\delta_{i, n_{i}},
\end{aligned}
$$

where

$$
\begin{aligned}
& \eta_{i, n_{i}}=\min \left(4^{1 / 3}\left(k_{i, n_{i}}-\frac{3}{4}\right), \frac{\sigma_{i, n_{i}}}{2^{1 / 3} \lambda_{\max }\left(\Gamma_{i, n_{i}}^{-1}\right)^{2 / 3}}\right), \\
& \delta_{i, n_{i}}=\frac{1}{4} \varepsilon_{i, n_{i}}^{4}+\sigma_{i, n_{i}} W_{i, n_{i}}^{(2 / 3) * T} W_{i, n_{i}}^{(2 / 3) *} .
\end{aligned}
$$

Then, the virtual control can be got as

$$
v=-z_{i, n_{i}}-k_{i, n_{i}+1} z_{i, n_{i}+1}-\widehat{W}_{i, n_{i}+1}^{T} \Psi_{i, n_{i}+1}\left(Z_{i, n_{i}+1}(t)\right) .
$$

Choose Lyapunov candidate functions

$$
V_{i}=\sum_{j=1}^{n_{i}+1} V_{i, j} .
$$

Then, based on (24), (37), and (51), it has

$$
\dot{V}_{i} \leq-\eta_{i} V_{i}^{2 / 3}+\delta_{i}
$$

where

$$
\begin{aligned}
\eta_{i} & =\min \left(\eta_{i, j}\right), \quad j=1,2,3, \ldots, n_{i}+1, \\
\delta_{i} & =\sum_{j=1}^{n_{i}+1} \delta_{i . j},
\end{aligned}
$$

Theorem 1. Consider the nonlinear system for which the model dynamics is approximated by neural networks (12), (27), and (40), the control law (53) with the virtual control (14), (29), and (42), and adaptive laws (15), (30), and (43); then, the following statements hold:

(1) All the signals of the closed-loop system, including $x_{i, j}, \alpha_{i, j}, \widehat{W}_{i, j}$, remain bounded all the time. 
(2) The closed-loop signal $z_{i, j}$ converge to a compact set defined by

$$
\Omega_{i, s}=\left\{z_{i} \mid\left\|z_{i}\right\|^{2} \leq \frac{\delta_{i}}{\eta_{i}}\right\},
$$

(i) where $\delta_{i}, \eta_{i}$ are constants related to the design parameters.

(3) The finite-time $T$ is given by

$$
T \leq \frac{3 V^{1 / 3}\left(x_{0}\right)}{\eta \theta_{0}},
$$

where $\eta, \theta_{0}$ are constants related to the design parameters and $V\left(x_{0}\right)$ is constant related to Lyapunov candidate functional.

Proof. considering Lyapunov function candidate (54) and (55), then $V_{i}$ is bounded. Therefore, it can conclude that for all $1 \leq j \leq n_{i}+1$, the error signals $z_{i, j}, \widetilde{W}_{i, j}$ are stable.

\section{Simulation Example}

In this section, simulation example shows the validity and feasibility of the proposed NN finite adaptive control design approach. Figure 1 shows the topology of communication graph of MAS with one leader and five followers.

Consider the following MAS with adjacency matrix $A$ and Laplacian matrix $L$ as follows:

$$
\begin{aligned}
A & =\left[\begin{array}{lllll}
0 & 0 & 0 & 0 & 3 \\
1 & 0 & 0 & 0 & 0 \\
0 & 3 & 0 & 0 & 0 \\
0 & 0 & 2 & 0 & 0 \\
0 & 0 & 0 & 1 & 0
\end{array}\right], \\
L & =\left[\begin{array}{ccccc}
3 & 0 & 0 & 0 & -3 \\
-1 & 1 & 0 & 0 & 0 \\
0 & -3 & 3 & 0 & 0 \\
0 & 0 & -2 & 2 & 0 \\
0 & 0 & 0 & -1 & 1
\end{array}\right] .
\end{aligned}
$$

The dynamic systems are described by

$$
\begin{aligned}
\dot{x}_{i, 1}= & 0.5 \sin \left(x_{i, 1}\right)-x_{i, 1}+\sin \left(x_{i, 2}\right)+x_{i, 2} \\
& +5 \sin (t)+5 \cos (t), \\
\dot{x}_{i, 2}= & -\sin \left(x_{i, 2}\right)-i x_{i, 2}+x_{i, 2} u_{i}+u_{i}+\sin \left(u_{i}\right), \\
y_{i}= & x_{i, 1} .
\end{aligned}
$$

$y_{d}=5 \sin (t)$ is the state, control input, control output, and ideal output, respectively. The control objective of the proposed design method is to make the system output $y$ follow the desired reference signal $y_{d}=5 \sin (t)$. Select the controller $\dot{u}$ and virtual control $\alpha_{1}, \alpha_{2}$ as follows:

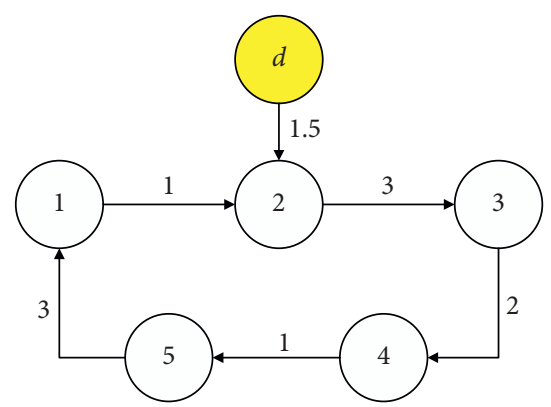

FIgURE 1: Topology of communication graph.

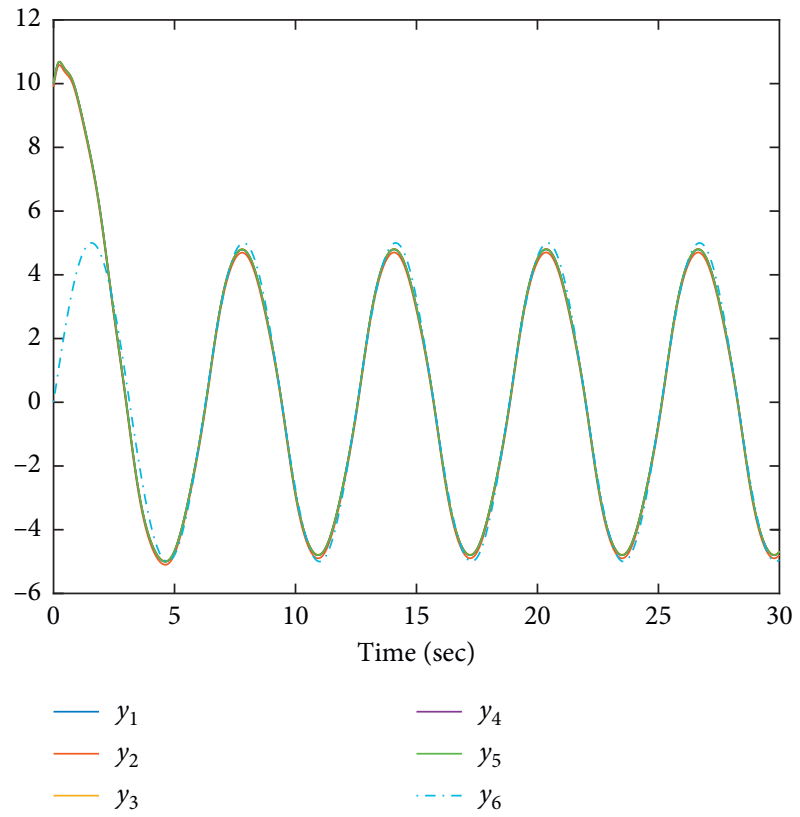

Figure 2: Output trajectory of five followers and leader.

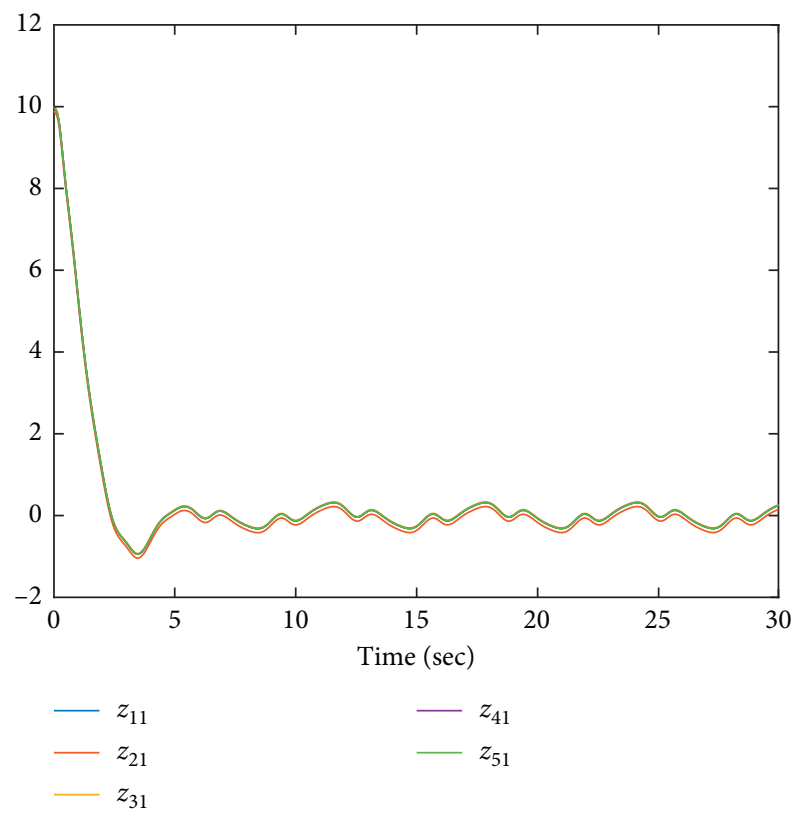

FIGURE 3: Error trajectory of five followers. 


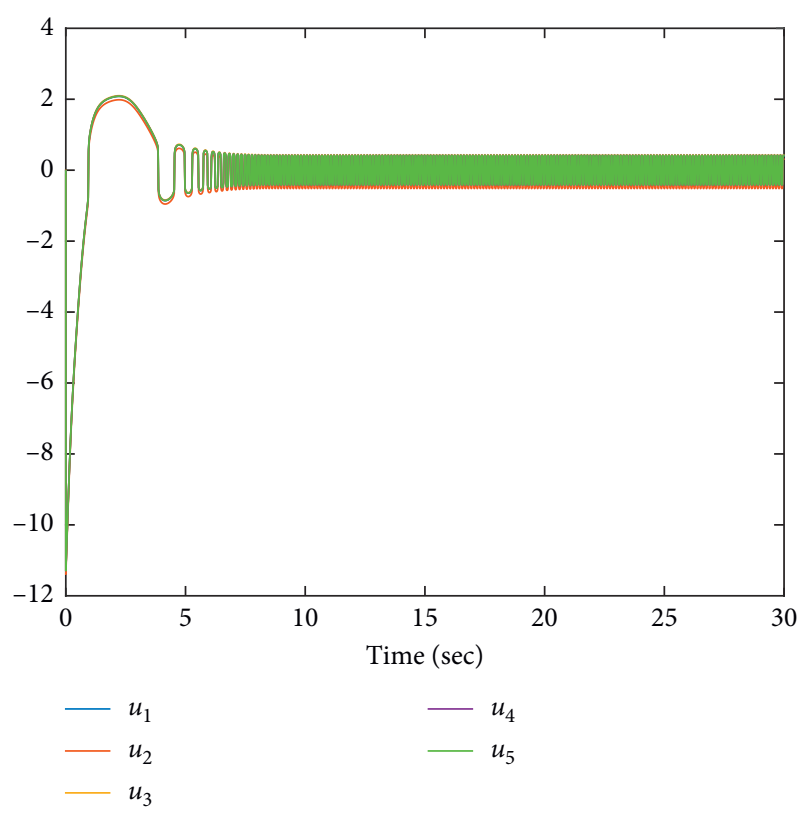

FIGURE 4: Five control trajectory.

$$
\begin{aligned}
\dot{u}_{i} & =-z_{i, 2}-k_{3} z_{i, 3}^{1 / 3}-\widehat{W}_{i, 3}^{T} \Psi_{3}\left(\bar{x}_{i, 2}, u_{i}\right), \\
\alpha_{i, 1} & =-k_{1} z_{i, 1}^{1 / 3}+x_{i, 2}-\widehat{W}_{i, 1}^{T} \Psi_{1}\left(\bar{x}_{i, 2}\right), \\
\alpha_{i, 2} & =-z_{i, 1}-k_{2} z_{i, 2}^{1 / 3}+u_{i}-\widehat{W}_{i, 2}^{T} \Psi_{2}\left(\bar{x}_{i, 2}, u_{i}\right) .
\end{aligned}
$$

The weight of NNs is updated by

$$
\dot{\hat{W}}_{i, j}=\Gamma_{i, j}\left[z_{i, j} \Psi_{i}\left(\bar{x}_{i, j+1}\right)-\sigma_{i, j} \widehat{W}_{i, j+1}^{1 / 3}\right], \quad j=1,2,3,
$$

with $z_{i, 1}=x_{i, 1}-y_{i, d}, z_{i, 2}=x_{i, 2}-\alpha_{i, 1}$, and $z_{i, 3}=u_{i}-\alpha_{i, 2}$.

Applying the finite-time NN control method, the simulation results are shown in Figures 2-4. Figure 2 shows the trajectory of output and desired reference trajectory of MAS. Figure 3 shows the error trajectory of five followers. Figure 4 shows the trajectory of controller. The method in this paper is more generally used, and both output and weight of NNs are convergence in finite time.

\section{Conclusion}

In this article, the adaptive tracking control method is proposed for distributed multiagent systems in nonaffine form. Based on finite neural network algorithm, a finite-time tracking result can be got. In addition, each follower agent is modeled by a nonlinear pure-feedback system with nonaffine form, and the nonlinear system is unknown functions rather than constants. Simulations and theoretical analysis are carried out to verify the feasibility and correctness of the proposed method.

\section{Data Availability}

No data were used to support this study.

\section{Conflicts of Interest}

The authors declare that there are no conflicts of interest regarding the publication of this paper.

\section{Acknowledgments}

This paper was supported by the 2018 Social and Science Fund (HB18GL075) and 2015 Hebei Province Natural Science Fund Project (G2015203378 and F2015208128).

\section{References}

[1] B. Burmeister, A. Haddadi, and G. Matylis, "Application of multi-agent systems in traffic and transportation," IEE Proceedings - Software Engineering, vol. 144, no. 1, pp. 51-60, 1997.

[2] J. Clerc and G. J. Wiens, "Reconfigurable multi-agent robots with mixed modes of mobility," in Proceedings of the IEEE International Conference on Robotics and Automation, Sendai, Japan, April 2004.

[3] C. Ramos, "An architecture and a negotiation protocol for the dynamic scheduling of manufacturing systems," in Proceedings of the 1994 IEEE International Conference on Robotics and Automation, San Diego, CA, USA, May 1994.

[4] N. R. Jennings, L. Z. Varga, R. P. Aarnts, J. Fuchs, and P. Skarek, "Transforming standalone expert systems into a community of cooperating agents," Engineering Applications of Artificial Intelligence, vol. 6, no. 4, pp. 317-331, 1993.

[5] H. Du, J. Zhou, D. Wu, and G. Wen, "Consensus for secondorder nonlinear leader-following multi-agent systems via event-triggered control," in Proceedings of the 2017 International Workshop on Complex Systems and Networks (IWCSN), Doha, Qatar, December 2017.

[6] K. Chen, J. Wang, and Y. Zhang, "Adaptive leader-following consensus of nonlinear multi-agent systems with jointly connected topology," in Proceedings of the the 27th Chinese Control and Decision Conference (2015 CCDC), Qingdao, China, May 2015.

[7] S. Ali, S. Ahmed, and S. N. K. Marwat, "A practical approach to consensus based control of multi-agent systems," in Proceedings of the 2018 International Symposium on Recent Advances in Electrical Engineering (RAEE), Islamabad, Pakistan, October 2018.

[8] L. Xu, J. Zheng, N. Xiao, and L. Xie, "Mean square consensus of multi-agent systems over fading networks with directed graphs," Automatica, vol. 95, pp. 503-510, 2018.

[9] Y. Cheng, T. Hu, Y. Li, and S. Zhong, "Consensus of fractional-order multi-agent systems with uncertain topological structure: A Takagi-Sugeno fuzzy event-triggered control strategy," Fuzzy Sets and Systems, 2020.

[10] Z. Li, G. Wen, Z. Duan, and W. Ren, "Designing fully distributed consensus protocols for linear multi-agent systems with directed graphs," IEEE Transactions on Automatic Control, vol. 60, no. 4, pp. 1152-1157, 2015.

[11] L. Ping, Q. Kaiyu, and S. Mengji, "Distributed robust $H_{\infty}$ rotating consensus control of multi-agent systems with mixed uncertainties and time-delay," in Proceedings of the Proceedings of the 32nd Chinese Control Conference, Xi' an, China, July 2013.

[12] K. Liu, H. Zhu, and J. Lu, "Robust consensus of a class of linear multi-agent systems via sampled-data control," in Proceedings of the 2015 10th Asian Control Conference (ASCC), Kota Kinabalu, Malaysia, June 2015. 
[13] J. Liu, Z. Liu, and Z. Chen, "Robust Ho consensus control for multi-agent system with dynamic topologies and time-varying delays," in Proceedings of the 29th Chinese Control Conference, Beijing, China, July 2010.

[14] D. Han, G. Chesi, and Y. S. Hung, "Robust consensus for a class of uncertain multi-agent dynamical systems," IEEE Transactions on Industrial Informatics, vol. 9, no. 1, pp. 306-312, 2013.

[15] C. Lin, W. Huang, Y. Huang, and S. Chen, "Robust consensus for linear multi-agent systems over randomly switching topologies," in Proceedings of the 2019 Chinese control conference (CCC), Wuhan, China, July 2019.

[16] J. Wu, D. Li, D. Lao et al., "Second-order robust consensus for nonlinear multi-agent systems with extended state observer," in Proceedings of the 33rd Chinese Control Conference, Nanjing, China, July 2014.

[17] P. Gong, "Distributed robust adaptive consensus control for uncertain nonlinear fractional-order multi-agent systems," in Proceedings of the 2019 Chinese Control and Decision Conference (CCDC), Nanchang, China, June 2019.

[18] X. Long, K. Zhou, and J. Hou, "Finite-time consensus for second-order multi-agent systems with disturbances by integral sliding mode algorithm based on relative information," in Proceedings of the 2018 37th Chinese control conference (CCC), Wuhan, China, July 2018.

[19] B. Luo, Q. Li, and Y. Yang, "Finite-time consensus for multiagent systems via sinusoidal controller," in Proceedings of the 2012 International Conference on Control Engineering and Communication Technology, Shenyang, China, December 2012.

[20] Y. Zhu, C. Chen, and X. Guan, "Finite-time consensus for multi-agent systems via impulsive control," in Proceedings of the 2016 35th Chinese Control Conference (CCC), Chengdu, China, July 2016.

[21] J. Liu, Y. Zhang, Y. Yu, and C. Sun, "Fixed-time event-triggered consensus for nonlinear multiagent systems without continuous communications," IEEE Transactions on Systems, Man, and Cybernetics: Systems, vol. 49, no. 11, pp. 2221-2229, 2019.

[22] J. Liu, Q. Wang, and Y. Yu, "Fixed-time consensus algorithm for second-order multi-agent systems with bounded disturbances," in Proceedings of the 2016 31st Youth Academic Annual Conference of Chinese Association of Automation (YAC), Wuhan, China, November 2016.

[23] J. Ni, L. Liu, C. Liu, and J. Liu, "Fixed-time leader-following consensus for second-order multiagent systems with input delay," IEEE Transactions on Industrial Electronics, vol. 64, no. 11, pp. 8635-8646, 2017.

[24] W. Zhang, X. Gao, B. Sun, X. Hu, and K. Zhu, "Echo state network for extended state observer and sliding mode control of vehicle drive motor with unknown hysteresis nonlinearity," Mathematical Problems in Engineering, vol. 2020, pp. 1-13, Article ID 2534038, 2020.

[25] J. H. Park, "Exponential stability of antiperiodic solution for BAM neural networks with time-varying delays," Mathematical Problems in Engineering, vol. 2018, Article ID 3034794, , 2018.

[26] J. Zhang, Q. Zhu, and Y. Li, “Convergence time calculation for supertwisting algorithm and application for nonaffine nonlinear systems," Complexity, vol. 2019, pp. 1-15, Article ID 6235190, 2019.

[27] J. Zhang, Q. Zhu, Y. Li, and X. Wu, "Homeomorphism mapping based neural networks for finite time constraint control of a class of nonaffine pure-feedback nonlinear systems," Complexity, vol. 2019, pp. 1-11, Article ID 9053858 , 2019.

[28] J. Zhang, Y. Li, W. Fei, and X. Wu, " $U$-model based adaptive neural networks fixed-time backstepping control for uncertain nonlinear system," Mathematical Problems in Engineering, vol. 2020, pp. 1-7, Article ID 8302627, 2020. 